\title{
Fatores associados à assistência pré-natal entre mulheres de baixa renda no Estado de São Paulo, Brasil *
}

\author{
Factors associated with pre-natal care among low income women, State of \\ S.Paulo, Brazil
}

Maria José Duarte Osis**, Ellen Hardy**, Aníbal Faúndes**, Graciana Alves**

\begin{abstract}
OSIS, M. J. D. et al. Fatores associados à assistência pré-natal entre mulheres de baixa renda no Estado de São Paulo, Brasil. Rev. Saúde Pública, 27:49-53, 1993. São apresentados resultados de pesquisa que avaliou o Programa de Assistência Integral à Saúde da Mulher (PAISM), realizada em 1988, no Estado de São Paulo, Brasil. Foram entrevistadas 3.703 mulheres de baixa renda que tinham entre 15 e 49 anos de idade, utilizando um questionário estruturado e pré-testado. Os resultados referem-se às 669 mulheres grávidas durante 1987 ou 1988 que responderam às questões sobre assistência pré-natal, parto e puerpério. Foi analisada a associação entre algumas de suas características sociodemográficas e comparecimento às consultas pré-natais, a idade gestacional em que foi feita a primeira consulta e o número total de consultas. Os resultados mostraram associação entre características sociodemográficas e comparecimento ao pré-natal. A maior percentagem de grávidas que fizeram pré-natal tinham mais que o primeiro grau de escolaridade. Foi maior a proporção de mulheres que começaram o pré-natal até o terceiro mês de gravidez entre aquelas que não tinham filho vivo (74\%), que viviam com um companheiro (70\%), que tirham mais que o primeiro grau de escolaridade $(88 \%)$ e as que moravam no interior do Estado $(71 \%)$.
\end{abstract}

Descritores: Cuidado pré-natal, utilização. Fatores socioeconômicos. Idade gestacional.

\section{Introduçáo}

A assistência pré-natal tem como objetivos principais assegurar uma evolução normal da gravidez; preparar a mãe para um parto, puerpério e lactação normais; identificar o mais rápido possível as situaçðes de risco, para que seja possível prevenir as complicaçóes mais freqüentes da gravidez e do ciclo puerperal ${ }^{9}$.

Diversos estudos descrevem a associação entre a assistência pré-natal e os resultados perinatais, particularmente o peso ao nascer, mortalidade perinatal e até a mortalidade infantil. O comparecimento às consultas de pré-natal e o seu número total têm apresentado correlação positiva significa-

\footnotetext{
* Parcialmente financiado pela Secretaria de Estado da Saúde do Estado de São Paulo.

* Centro de Pesquisas e Controle das Doenças MatemoInfantis de Campinas (CEMICAMP) - Campinas, SP Brasil.

*** CEMICAMP e Departamento de Tocoginecologia. Faculdade de Ciências Médicas, Universidade Estadual de Campinas (UNICAMP) - Campinas, SP - Brasil.
}

Separatas/Reprints: E. Hardy - Caixa Postal 6181 - 13081-970 Campinas, SP - Brasil.

Edição subvencionada pela FAPESP. Processo Medicina 93/ 0208-5. tiva com o peso ao nascer e com a probabilidade de uma mulher ter um recém-nascido de peso nor$\mathrm{mal}^{7}, 8,12,14$.

A literatura também tem identificado associação significativa entre mortalidade perinatal e o número de consultas de pré-natal em dois países da América Latina, incluindo o Brasi13,8. Não fica claro, entretanto, se essa é uma relação direta ou indireta, devido à associação do baixo nível socioeconômico tanto com maus resultados perinatais como com falta de pré-natal.

Há evidências na literatura de associação entre classe social e mortalidade perinatal e prematuridade $2,10,16$, e, ainda, com morbi-mortalidade infantil $^{4,5,13}$. Caldwell ${ }^{5}$, em estudo de dados nigerianos, deu particular importância à escolaridade materna como rator determinante de menor mortalidade infantil. A influência da escolaridade permanece mesmo após ser controlada por outras variáveis sociais e pode ser independente da qualidade dos cuidados recebidos durante a gestação.

Não há definição clara de como classe social baixa ou aspectos socioeconômicos influenciam os resultados perinatais. Entretanto, há algumas indicações de que a nutrição, a estatura e o trabalho da mãe durante a gravidez são fatores associados a tais resultados ${ }^{8}$.

A diferença no tratamento médico durante 0 
pré-natal e o pós-parto pode ser também componente importante nessa associação.

Por outro lado, a influência do pré-natal sobre os resultados perinatais tem-se mantido depois de controlada por alguns fatores socioeconômicos como escolaridade; e além disso, a influência da escolaridade sobre os resultados perinatais tende a desaparecer quando a gestante consegue bons cuidados pré-natais ${ }^{8}$.

A importância da assistência pré-natal levou a Secretaria de Saúde do Estado de São Paulo a se preocupar com as deficiências nessa área, constatadas no Estado: em 1981, 30\% das mulheres com baixa renda não fizeram pré-natal comparadas com somente $5 \%$ entre as de classe média ou alta ${ }^{11}$. Este problema foi uma das razões para pretender implantar, no Estado, o Programa de Assistência Integral à Saúde da Mulher (PAISM), já que um dos seus objetivos é justamente melhorar a cobertura e a qualidade da assistência pré-natal.

Para avaliar algumas das atividades desenvolvidas pelo PAISM, no Estado de São Paulo, foi feito um estudo, em 1988, na área metropolitana da cidade de São Paulo e no interior. A pesquisa permitiu identificar as principais deficiências do sistema tanto na cobertura como na qualidade do atendimento ${ }^{15}$.

$O$ presente trabalho apresenta resultados desse estudo referentes à assistência pré-natal. No grupo de mulheres que estavam grávidas em 1987/88 foi analisada a associação entre algumas de suas características sociodemográficas e comparecimento aos cuidados pré-natais, a idade gestacional em que foi feita a primeira consulta e o número total de consultas.

\section{Material e Método}

O estudo foi feito em 1988 em nove dos 62 então chamados Escritórios Regionais de Saúde (ERSAs) do Estado de São Paulo, que correspondem aos atuais Sistemas Unificados e Descentralizados de Saúde (SUDS). Esses órgãos da Secretaria Estadual de Saúde gerenciam a execução dos serviços que estão sob sua responsabilidade, provendo-os com seus próprios recursos ou através de articulação inter-institucional. Cada ERSA inclui vários municípios ou bairros, como na área metropolitana de São Paulo. Foram entrevistadas, em seus próprios domicílios, 3.703 mulheres de baixa renda que tinham entre 15 e 49 anos de idade. Para as entrevistas, foi utilizado um questionário estruturado e pré-testado.

As mulheres que responderam às questð̃es sobre assistência pré-natal, parto e puerpério foram as 669 que estiveram grávidas durante 1987 ou 1988.
Para a análise dos dados foram usados os seguintes pacotes estatísticos: Statistical Package for the Social Sciences (SPSS/PC) e Statistical Analysis System (SAS). As diferenças entre os grupos foram avaliadas com o teste de qui-quadrado ${ }^{1}$. A regressão $\operatorname{logística~}^{6}$ foi usada para identificar os fatores associados a ter feito pré-natal, a tê-lo iniciado até o terceiro mês de gravidez e ter feito, no total, seis ou mais consultas.

A regressão logística foi usada para identificar os fatores associados a ter feito pré-natal, tê-lo iniciado até o terceiro mês de gravidez e ter feito seis ou mais consultas no total. Todas as variáveis mostradas nas Tabelas 1, 2 e 3 foram incluídas no modelo.

\section{Resultados}

Houve uma maior percentagem de grávidas que fizeram pré-natal entre aquelas que tinham mais que o primeiro grau de escolaridade (95\%), comparadas com as sem escolaridade ou que completaram no máximo o primeiro grau (85\%). Esta diferença foi estatisticamente significativa (Tabela 1). Não houve diferenças significativas na percenta-

Tabela 1. Grávidas que fizeram pré-natal, segundo algumas características sociodemográficas (em percentagem). Estado de São Paulo, 1987-88.

\begin{tabular}{|c|c|c|c|c|}
\hline Características & Pré-natal & $N$ & $x^{2}$ & $P$ \\
\hline $\begin{array}{l}\text { Idade na última gravidez } \\
\text { Até } 19 \\
20-29 \\
30 \text { ou mais }\end{array}$ & $\begin{array}{l}83,5 \\
88,3 \\
85,0\end{array}$ & $\begin{array}{l}103 \\
411 \\
153\end{array}$ & 2,3 & n.s. \\
\hline $\begin{array}{l}\text { Filhos vivos na entrevista } \\
0 \\
1 \\
2-3 \\
4 \text { ou mais }\end{array}$ & $\begin{array}{l}85,9 \\
87,9 \\
88,3 \\
77,8\end{array}$ & $\begin{array}{r}85 \\
214 \\
298 \\
72\end{array}$ & 5,9 & n.s. \\
\hline $\begin{array}{l}\text { Estado marital } \\
\text { Com companheiro } \\
\text { Sem companheiro }\end{array}$ & $\begin{array}{l}87,2 \\
83,7\end{array}$ & $\begin{array}{r}577 \\
92\end{array}$ & 0,8 & n.s. \\
\hline $\begin{array}{l}\text { Escolaridade } \\
\text { Até } 1^{\circ} \text { grau"* } \\
\text { Mais que } 1^{\circ} \text { grau }\end{array}$ & $\begin{array}{l}84,8 \\
94,6\end{array}$ & $\begin{array}{l}540 \\
129\end{array}$ & 8,6 & $<0,004$ \\
\hline $\begin{array}{l}\text { Trabalho pago } \\
\text { Sim } \\
\text { Não }\end{array}$ & $\begin{array}{l}90,4 \\
85,5\end{array}$ & $\begin{array}{l}166 \\
503\end{array}$ & 2,6 & n.s. \\
\hline $\begin{array}{l}\text { Região } \\
\text { Área metropolitana } \\
\text { Interior }\end{array}$ & $\begin{array}{l}84,5 \\
89,5\end{array}$ & $\begin{array}{l}381 \\
288\end{array}$ & 3,7 & n.s. \\
\hline
\end{tabular}


gem de grávidas que compareceram ao pré-natal segundo idade, número de filhos vivos, estado marital, trabalho e região de residência.

Foi significativamente maior a proporção de mulheres que começaram o pré-natal até o terceiro mês de gravidez entre aquelas que não tinham nenhum filho vivo $(74 \%)^{*}$; aquelas que viviam com um companheiro (70\%); aquelas que tinham mais que o primeiro grau de escolaridade $(88 \%)$, e aquelas que moravam no interior do Estado (71\%). Não houve diferenças segundo idade e trabalho da mulher (Tabela 2).

Tabela 2. Grávidas que fizeram a primeira consulta de pré-natal até o terceiro mês de gravidez, segundo algumas características sociodemográficas (em percentagem). Estado de São Paulo, 1987-88.

\begin{tabular}{|c|c|c|c|}
\hline Características & $\begin{array}{c}\text { Primeira } \\
\text { consulta até } \\
3^{\circ} \text { mês }\end{array}$ & $\mathbf{N}$ & $\mathbf{P}$ \\
\hline $\begin{array}{l}\text { Idade na última gravidez } \\
\text { Até } 19 \\
20-29 \\
30 \text { ou mais }\end{array}$ & $\begin{array}{l}64,1 \\
67,6 \\
68,0\end{array}$ & $\begin{array}{l}103 \\
411 \\
153\end{array}$ & n.s. \\
\hline $\begin{array}{l}\text { Filhos vivos na entrevista } \\
0 \\
1 \\
2-3 \\
4 \text { ou mais }\end{array}$ & $\begin{array}{l}74,1 \\
72,4 \\
66,4 \\
45,8\end{array}$ & $\begin{array}{r}85 \\
214 \\
298 \\
72\end{array}$ & $<0,0003$ \\
\hline $\begin{array}{l}\text { Estado marital } \\
\text { Com companheiro } \\
\text { Sem companheiro }\end{array}$ & $\begin{array}{l}69,8 \\
50,0\end{array}$ & $\begin{array}{r}577 \\
92\end{array}$ & $<0,0003$ \\
\hline $\begin{array}{l}\text { Escolaridade } \\
\text { Até } 1^{2} \text { grau** } \\
\text { Mais que } 1^{\circ} \text { grau }\end{array}$ & $\begin{array}{l}62,0 \\
88,4\end{array}$ & $\begin{array}{l}540 \\
129\end{array}$ & $<0,0001$ \\
\hline $\begin{array}{l}\text { Trabalho pago } \\
\text { Sim } \\
\text { Não }\end{array}$ & $\begin{array}{l}72,3 \\
65,4\end{array}$ & $\begin{array}{l}166 \\
503\end{array}$ & n.s. \\
\hline $\begin{array}{l}\text { Região } \\
\text { Área metropolitana } \\
\text { Interior }\end{array}$ & $\begin{array}{l}63,8 \\
71,5\end{array}$ & $\begin{array}{l}381 \\
288\end{array}$ & $<0,04$ \\
\hline
\end{tabular}

- Faltou informação de duas mulheres na área metropolitana.

** As mulheres sem escolaridade foram incluidas.
As mulheres que fizeram seis ou mais consultas de pré-natal foram principalmente aquelas que tinham um filho vivo por ocasião da entrevista $(61 \%)$ e as com dois ou três $(58 \%)$; que viviam com um companheiro (56\%); que tinham maior escolaridade (68\%) e que moravam no interior do Estado (59\%). Essas diferenças foram estatisticamente significativas. Com relação à idade na última gravidez e trabalho pago não houve diferenças estatísticas entre os grupos (Tabela 3).

Tabela 3. Grávidas que fizeram seis ou mais consultas de pré-natal*, segundo algumas características sociodemográficas (em percentagem). Estado de São Paulo, 1987-88.

\begin{tabular}{|c|c|c|c|}
\hline Caracteristicas & $\begin{array}{c}\text { Seis ou } \\
\text { mais } \\
\text { consultas }\end{array}$ & $\mathbf{N}$ & $P$ \\
\hline $\begin{array}{l}\text { Idade na última gravidez"* } \\
\text { Até } 19 \\
20-29 \\
30 \text { ou mais }\end{array}$ & $\begin{array}{l}47,0 \\
54,5 \\
57,6\end{array}$ & $\begin{array}{l}100 \\
404 \\
151\end{array}$ & n.s. \\
\hline $\begin{array}{l}\text { Filhos vivos na entrevista } \\
0 \\
1 \\
2-3 \\
4 \text { ou mais }\end{array}$ & $\begin{array}{l}33,3 \\
60,7 \\
57,9 \\
41,4\end{array}$ & $\begin{array}{r}84 \\
211 \\
292 \\
70\end{array}$ & $<0,0003$ \\
\hline $\begin{array}{l}\text { Estado marital } \\
\text { Com companheiro } \\
\text { Sem companheiro }\end{array}$ & $\begin{array}{l}56,5 \\
37,4\end{array}$ & $\begin{array}{r}566 \\
91\end{array}$ & $<0,0008$ \\
\hline $\begin{array}{l}\text { Escolaridade } \\
\text { Até } 1^{2} \text { grau*** } \\
\text { Mais que } 1^{2} \mathrm{grau}\end{array}$ & $\begin{array}{l}50,6 \\
67,7\end{array}$ & $\begin{array}{l}530 \\
127\end{array}$ & $<0,0006$ \\
\hline $\begin{array}{l}\text { Trabalho pago } \\
\text { Sim } \\
\text { Não }\end{array}$ & $\begin{array}{l}58,4 \\
52,3\end{array}$ & $\begin{array}{l}166 \\
491\end{array}$ & n.s. \\
\hline $\begin{array}{l}\text { Região } \\
\text { Área metropolitana } \\
\text { Interior }\end{array}$ & $\begin{array}{l}50,0 \\
58,9\end{array}$ & $\begin{array}{l}372 \\
285\end{array}$ & $<0,03$ \\
\hline
\end{tabular}

- 12 mulheres não lembravam o número de consultas.

* Faltou informação de duas mulheres na área metropolitana.

*** As mulheres sem escolaridade foram incluidas.

Tabela 4. Fatores associados a ter feito pré-natal, iniciano até o terceiro mês de gestação e feito seis ou mais consultas.

\begin{tabular}{lcccccc}
\hline \multirow{2}{*}{ Variáveis } & \multicolumn{2}{c}{ Fazer pré-natal } & \multicolumn{2}{c}{ Início até $3 \mathrm{~m}$} & \multicolumn{2}{c}{$>5$ consultas } \\
& $\mathrm{p}<$ & $\mathrm{R}$ & $\mathrm{p}<$ & $\mathrm{R}$ & $\mathrm{p}<$ & $\mathrm{R}$ \\
\hline Mais que $1^{2}$ grau & 0,006 & 0,105 & 0,0001 & 0,158 & 0,002 & 0,098 \\
Com companheiro & NS & & 0,0002 & 0,125 & 0,002 & 0,099 \\
Morar no interior & NS & & 0,05 & 0,051 & 0,03 & 0,055 \\
Menos filhos vivos & NS & & 0,0005 & 0,112 & NS & \\
\hline
\end{tabular}

* Refere-se a mulheres que alguma vez ficaram grávidas, mas que no momento da entrevista não tinham nenhum filho vivo (inclui grávidas pela primeira vez, abortos, natimortos ou falecidos).
A maior escolaridade esteve associada a ter feito pré-natal, iniciá-lo até o terceiro mês e fazer seis ou mais consultas no total. Viver com um companheiro e morar no interior foram fatores que 
se mostraram associados a ter iniciado o pré-natal até o terceiro mês e ter comparecido a seis ou mais consultas. $O$ início mais precoce do pré-natal esteve também associado estatisticamente ao menor número de filhos vivos da gestante (Tabela 4).

\section{Discussáo}

Os resultados confirmam a forte associação entre características socioeconômicas e comparecimento ao pré-natal, particularmente no que se refere ao nivel de escolaridade da gestante. Este se associa tanto a fazer ou não controle pré-natal como ao cumprimento das normas, a respeito de início precoce e número de consultas, o que está de acordo com publicaçōes anteriores ${ }^{8}$.

Viver com companheiro fixo, em matrimônio legal ou não, foi importante indicador de início precoce e maior número de consultas, mas não se mostrou associado ao comparecimento ou não ao pre-natal. Pode-se postular que a mulher solteira, independentemente da sua escolaridade, demora mais em aceitar a gestação e tem menos conhecimentos acerca do que fazer em caso de ficar grávida, o que poderia explicar o início mais tardio das consultas pré-natais e, conseqüentemente, também o menor número de consultas.

O comparecimento mais precoce das gestantes com menos filhos pode ser atribuído tanto às maiores dificuldades da mulher com mais filhos em sair de casa, quanto à crença de que esta já tem experiência em estar grávida e não precisa apressar-se em consultar o médico.

A associação entre morar no interior e assistência mais precoce e maior número de consultas deve-se mais ao sistema de saúde que às características das grávidas. Maior proximidade do posto ao lugar de moradia, maiores facilidades de condução e possíveis diferenças na facilidade para obter atendimento, podem ser fatores que explicam esta associação.

O contraponto desses resultados indica as deficiências de cobertura e qualidade que ainda persistem na assistência pré-natal no Estado de São Paulo. Apesar da percentagem de grávidas que fizeram pré-natal ter sido alta nas duas regiōes estudadas, ainda restaram quase $15 \%$ que não fizeram sequer uma consulta. Além disso, um terço das mulheres iniciaram o atendimento após o terceiro mês de gestação, e quase a metade não chegou a fazer seis consultas no total, condiçōes consideradas não apropriadas para assegurar um bom acompanhamento. Esse grupo esteve constituído, principalmente, por mulheres com menos escolaridade, com mais filhos, solteiras e que viviam na área metropolitana de Săo Paulo. Elas exigem, portanto, um esforço adicional dos serviços de saúde no sentido de serem esclarecidas a respeito da importância de se fazer um pré-natal adequado.

Esse esforço deve anteceder a chegada da cliente ao posto de saúde e corresponde ao nivel mais básico de assistência, que se inicia na comunidade. Requer-se o desenvolvimento de um processo educativo voltado para as necessidades dessas mulheres, que seja capaz de alcançá-las. Concomitantemente, é indispensável assegurarlhes sempre o acesso fácil aos serviços públicos e empenhar-se no constante treinamento dos profissionais que devem atender a essa população, para que se amplie não só a cobertura mas também melhore a qualidade da assistência, que assegure o retorno da mulher às consultas e efetivamente melhore os resultados perinatais.

OSIS, M. J. D. et al. [Factors associated with pre-natal care among low income women, State of S.Paulo, Brazil]. Rev. Saúde Pública, 27:49-53, 1993. This paper presents the results of a study carried out in 1988 in the State of S. Paulo, with the purpose of evaluating the Program for Women's Integral Health Care. A total of 3.703 low income women of $15-49$ years of age were interviewed using a structured, pre-tested form. The data in this paper relate to 669 women who had been pregnant during 1987 or 1988 and who were asked about pre-natal, delivery and post-partum care. The association between some of their socio-demographic characteristics and the pre-natal care received, months pregnant at the time of first visit and total number of visits, were analysed. Results showed an association between socio-economic characteristics and pre-natal care received. The greatest percentage of pregnant women who had had pre-natal care was found among those with more than elementary education. The highest percentage of women who started pre-natal care before the third month of pregnancy was found among those who had no living children $(74 \%)$, were in union $(70 \%)$, had at least some high school education $(88 \%)$ and lived in the interior of the state $(71 \%)$. The results show that it is necessary to increase the coverage and quality of prenatal care so as to improve perinatal results.

Keywords: Prenatal care, utilization. Socieconomic factors. Gestational age.

\section{Referências BIbllográflcas}

1. ARMITAGE, P. \& BERRY, G. Statistical methods in medical research. 2nd ed. Oxford, Blackwell Scientific Publ., 1987.

2. BAIRD, D. The influence of social and economic factors on still births and neonatal deaths. J. Obstet. Gynaec. Brit. Emp., 52: 339, 1945.

3. BARROS, F. C.; VICTORA, C. G.; VAUGHAN, J. P.; ESTANISLAU, H. J. Perinatal mortality in southern Brazil: a population based study of 7392 births. Bull. World Health Organ., 65: 95-104, 1987. 
4. BEHM, H. \& PRIMANTE, D. A. Mortalidad en los primeros anos de vida en la America.Latina. Notas Pobl., 6: 23, 1978.

5. CALDWELL, J. C. Edur:tion as a factor in mortality decline. An examination of Nigeria data. Popul. Stud., 33: 395, 1979.

6. COX, R. K. The analysis of binary data. London, Methnenx, 1970.

7. DONALDSON, P. J. \& BILLY, J. G. O. The impact of prenatal care on birth weight: evidence from an international data set. Med. Care, 22: 177-88, 1984.

8. FAÚNDES, A.; HARDY, E.; DLAZ, J.; PINOTTI, J. A Association of marital status and years of schooling with perinatal outcome: the influence of pre-natal care as an intermediate variable. J. Perinat. Med., 10: 105$13,1982$.

9. FAUNDES, A.; PINOTTI, J. A.; CECATTI, J. G. Atendimento pré-natal: assistência obstétrica primária: quais as necessidades no Brasil. J. Bras. Med., 52(3): 38-54, 1987.

10. FEDERICK, J. \& ANDERSON, A. B. M. Factors associat- ed with spontaneous pre-term birth. Br. J. Obstet. Gynaec., 83: 342, 1976.

11. FUNDAÇÃO IBGE. Pesquisa nacional por amostragem domiciliar (PNAD), Rio de Janeiro, 1981.

12. GORTMAKER, S. L. The effects of perinatal care upon the health of the newbom. Am. J. Public. Health, 69: 653$60,1979$.

13. KINCALD, J. C. Social pathology of fetal infant loss. Br. Med.J., 1: 1057, 1965.

14. KINCALD, J. C. Perinatal care: organization and outcome. Lancet, 2: 777-8, 1986.

15. PINOTTI, J. A.; FAÚNDES, A; HARDY, E.; REBELLO, I; OSIS, M. J. D.; SOUZA, T. R.; MORAES, T. M. Avaliação da assistência ginecológica no Estado de São Paulo. Rev. Ginec. Obstet., 1: 7-21, 1990.

16. THOMSON, A. M. Prematurity: socio-economic and nutritional factors. Mod. Prob. Pediat., 8: 197, 1963.

Recebido para publicaçäo em 26.6.1992 Reapresentado em 17.11.1992 Aprovado para publicaçäo em 19.11.1992 